seen outside the cells, but are especially characteristic when on the epithelial cells.

Smears from scarlet fever throats show many polymorphonuclear leukocytes and a variable number of cocci, usually round, in pairs, or chains of rarely more than four, with generally a wide capsule.

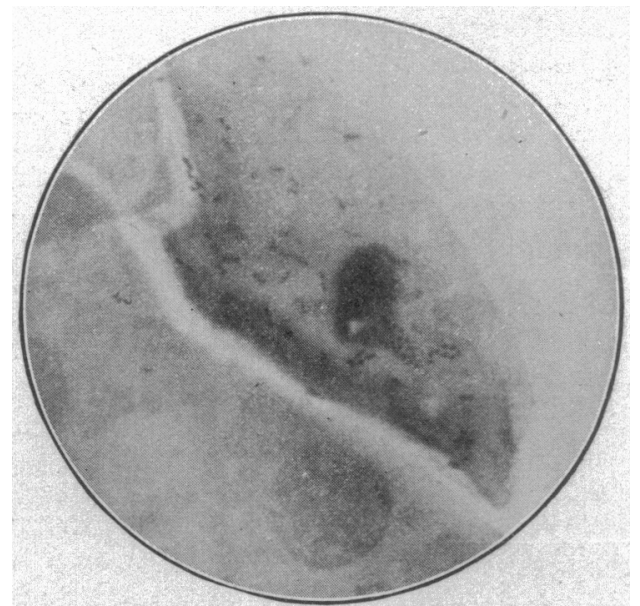

Fig. 2.-Smear from rubella throat; carbol-gentian violet; $\times 1,000$.

I find that the hemolytic streptococci isolated from the throats of scarlet fever patients as a rule show a capsule, at least in the first generation. This is in accord with the observations of Smillie, ${ }^{2}$ who found that freshly isolated hemolytic streptococci had a "capsular substance," disappearing rapidly on cultivation. He does not consider it a true capsule. The encapsulated diplococci in the smears are readily differentiated from cocci with a clear zone of retraction.

It has not been possible to distinguish the diplococcus in the scarlet fever throat smears from those in nonscarlatinal throats containing hemolytic streptococci (diphtheria, streptococcus sore throat, and measles). In a case of rubella following scarlet fever, both types of diplococci were observed in the throat smears.

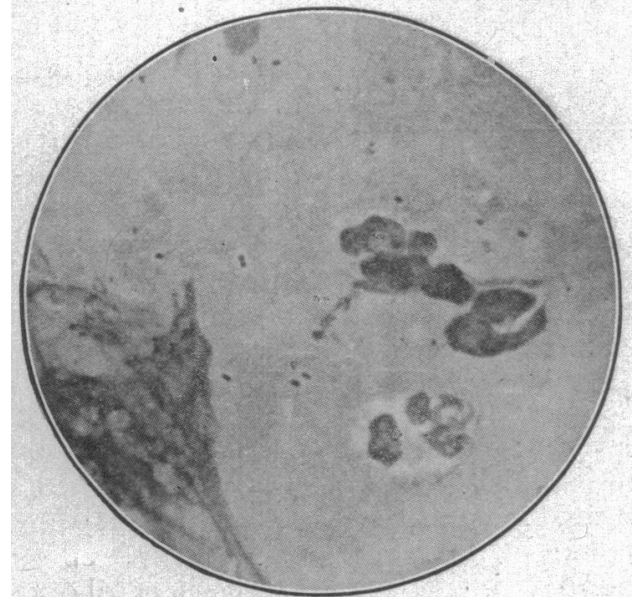

Fig. 3.-Smear from scarlet fever throat; carbol-gentian violet;

If the tonsils contain crypts so that a great variety of bacteria are present, it may be impossible to recognize any characteristics pointing to the source of the smear. A successful smear from the diagnostic point of view must contain one predominating diplococcus.

2. Smillie, W. G.: Jour. Infect. Dis., 1917, 20, 45.
The three types of diplococci described were not recognized in the thirty normal throats, except four times, when the rubella coccus was found in persons closely associated with rubella patients. The rubella diplococcus was also observed in three of the eight cases of so-called simple sore throat, two in roommates and one in a nurse of rubella patients. These smears were made during an epidemic of rubella, and doubtless the other diplococci can be found in smears of normal throats during epidemics of measles and scarlet fever.

There is rarely much difficulty in making a diagnosis of measles, but at times it is not easy to differentiate a mild case of scarlet fever from rubella. In the Durand Hospital, throat smears are proving helpful in differentiating these two diseases, especially in conjunction with leukocyte counts, as the number of leukocytes is increased in scarlet fever and reduced in rubella.

\section{RUPTURE OF THE UTERUS THROUGH THE CESAREAN SECTION SCAR *}

\author{
EMIL NOVAK, M.D. \\ BALTIMORE
}

The number of cases of rupture of the uterus following cesarean section that had been reported up to 1913 was sixty-three, according to the exhaustive review of the literature published in that year by Findley. ${ }^{1}$ By 1916 the number had risen to seventyfour, ${ }^{2}$ and since then a number of other cases have been reported. The occurrence must, therefore, be looked on as relatively rare, so that it would seem to be worth while to put even a single case on record. Aside from the infrequency of rupture of the cesareanized uterus, I have been led to report the following case by certain rather unusual circumstances attending it, and also by its pertinence to the debate as to the correctness or incorrectness of the old dictum, "Once a cesarean, always a cesarean."

\section{REPORT OF CASE}

History.-A white woman, aged 19, had had a cesarean section performed, May 5, 1916, the indication having been intrapartum eclampsia. The operation was performed by one of the most competent obstetricians in Baltimore. The child was delivered dead, but the mother recovered from the operation and her convalescence was uneventful, except for a slight infection of the abdominal incision.

The patient enjoyed good health and menstruated regularly up to October, 1917. The last regular menstruation appeared, Oct. 13, 1917. Some months later the patient consulted Dr. B. O. McCleary, who diagnosed pregnancy. The course of this second pregnancy was normal in every way. At about the expected date of confinement, July 15, the patient went into labor, and was then transferred to the hospital. Her own statement as to this confinement is that there were only two or three typical labor pains, these being followed by severe and constant pain over the entire abdomen, associated with some rigidity of the abdominal walls. This diffuse pain persisted for three days, being accompanied by a fever ranging from 99 to $101.5 \mathrm{~F}$., and by a slightly accelerated puise, never higher than 100 . After the subsidence of the pain, on about the fourth day, the patient was able to get around fairly comfortably, but she remained in the hospital awaiting the reinauguration of labor. According to Dr. McCleary, no fetal heart sound was to be heard

* From the Gynecological Department of Johns Hopkins University.

1. Findley, P.: Am. Jour. Obst., 1916, 74, 411

2. Bell, J. N.: Am. Jour. Obst., 1916, 74, 950. 
after this spurious labor, nor did the patient perceive any fetal movements.

Examination.-About six weeks after the expected time of confinement, August 29, I saw the patient in consultation with Dr. McCleary. At this time the abdomen was enlarged to the size of a full term pregnancy, the walls being quite rigid, so that the fetal parts could not be mapped out. No fetal heart sounds were heard. On vaginal examination, I was surprised to find that no presenting part could be felt, the cervix being closed and fairly firm, not resembling in feel the cervix of pregnancy. In view of the rather clearcut history, and of the foregoing findings, only one diagnosis seemed possible - that of rupture of the uterus with extrusion of the fetus into the abdominal cavity.

Operation.-The operation was performed, September 1. A long incision was made paralleling that of the previous cesarean section. Just above the umbilicus a thick, spongy tissue was encountered, which, on extension of the incision, was found to be the placenta. A few centimeters above the placental area, the amniotic sac was entered. About 2 quarts of clear amniotic fluid escaped. A large, partly macerated fetus was found lying in the abdominal cavity in an oblique position, the head being above and to the right, under the dome of the diaphragm, and the extremities extending downward and to the left. The amniotic sac was intact, except where it had been incised on the opening of the abdomen. The uterus had undergone involution a f $\mathrm{ter}$ rupture, being about the size that one would expect six weeks postpartum. The anterior wall of the uterus had been split asunder through the line of the old incision, the rift extending from the fundus to about the level of the internal os. This is well shown in the accompanying illustration, which also shows the manner in which the placenta was turned out through the uterine gap, partly reimplanting itself later on the anterior parietal peritoneum. Both fallopian tubes were covered with light, sheetlike adhesions. The right ovary was thoroughly disorganized through cystic degeneration, being about the size of a pullet's egg.

A subtotal hysterectomy was performed, the left ovary,

which was normal, being conserved. On removal of the uterus, together with the attached placenta, umbilical cord and fetus, an enormous cavity was left. The walls of this were formed in a general way by the parietes anteriorly, and by the adherent coils of intestine above and behind. It was a beautiful illustration of foreign body encapsulation. The inside of the cavity was lined by tightly adherent amniotic membrane, which was removed where this was possible. The large cavity left on the removal of the fetus was then marsupialized, several large cigaret drains being introduced in various portions, and then brought out through the abdominal incision. The latter was closed in tiers in the usual manner, being reinforced by a number of interrupted silkworm-gut sutures. Recovery from the operation was uneventful, the patient being discharged from the hospital three weeks after the operation.

\section{COMMENT}

A striking feature of this case was the fact that the occurrence of uterine rupture was not associated with internal hemorrhage or with shock. This observation, while unusual, is not unique, a case similar in this respect having been recently reported by Neill. ${ }^{3}$ The uterine scar in my patient seems to have been separated without producing any hemorrhage whatsoever, perhaps because of the tampon-like action of the fetal head, as it was delivered through the gap. Certainly there was no sign of either old or recent hemorrhage at the subsequent laparotomy, nor was there any appreciable degree of shock at the time of the occurrence of the rupture. As has been stated, there was more or less diffuse abdominal pain for a few days, but after that the patient was comparatively comfortable, walking about the ward with little inconvenience.

From the standpoint of obstetric practice the most important feature of this case is furnished by the history of an infected abdominal incision, with slight fever for several days, after the cesarean section which had been performed in 1916. The investigations of Williams, ${ }^{4}$ Findley ${ }^{1}$ and others assign great significance to such an occurrence. It appears to have been pretty well demonstrated that uterine rupture a $f$ t e $r$ cesarean section practically never occurs when the convalescence from the cesarean operation has been entirely normal, that is, when there has been no febrile reaction or other evidence of infection of the uterine scar. In other words, assuming that the uterine wall has been properly sutured, rupture in subsequent pregnancies will not take place if the uterine incision heals promptly, without infection. The invasion of the uterine scar by decidual elements in subsequent pregnancies, to which some have attached importance as predisposing to rupture, is probably of much less importance than the element of infection. In my patient, the occurrence of stitch infection in the abdominal incision after the cesarean section may be taken as prima facie evidence of infection and poor healing of the uterine incision, which had been sutured by the best technic.

As far, therefore, as the bearing of the case on the general question is concerned, it emphasizes the ominous significance of infection in the uterine incision as an element of danger in subsequent pregnancy. On the other hand, nothing in the case shakes in any way the position held by most obstetricians that the management of cesarean patients in subsequent pregnancies should not be too strongly influenced by the fact that rupture of the uterus occurs in a small proportion of the patients, probably not exceeding 2 or 3 per cent.

26 East Preston Street.

3. Neill, T. E.: Am. Jour. Obst., 1917, 75, 235

4. Williams, J. W.: Bull. Johns Hopkins Hosp., 1917, 28, 335. 